\title{
Hypochlorous Acid Can Be the Novel Option for the Meibomian Gland Dysfunction Dry Eye through Ultrasonic Atomization
}

\author{
Zhiyuan Li $\mathbb{D}^{1,2}$ Haiyan Wang, ${ }^{3}$ Mo Liang, ${ }^{1,2}$ Zhenghua Li, ${ }^{1,2}$ Yvliang Li, ${ }^{1,2}$ \\ Xiaoping Zhou, ${ }^{1,2}$ and Guoping Kuang ${ }^{1,2}$ \\ ${ }^{1}$ The Diagnosis and Treatment Technology Research and Development Centre for Dry Eye and Ocular Surface Disease of Chenzhou, \\ Chenzhou, 423000 Hunan, China \\ ${ }^{2}$ The Ophthalmology Department of the Affiliated Chenzhou Hospital, Hengyan Medical School, University of South China, \\ Chenzhou, 423000 Hunan, China \\ ${ }^{3}$ The Operation Department of the Affiliated Chenzhou Hospital, Hengyan Medical School, University of South China, Chenzhou, \\ 423000 Hunan, China
}

Correspondence should be addressed to Zhiyuan Li; tolizhiyuan01@163.com

Received 22 October 2021; Revised 11 December 2021; Accepted 17 December 2021; Published 5 January 2022

Academic Editor: Ting Su

Copyright (c) 2022 Zhiyuan Li et al. This is an open access article distributed under the Creative Commons Attribution License, which permits unrestricted use, distribution, and reproduction in any medium, provided the original work is properly cited.

Background. Dry eye is a multifactor disease which needs comprehensive treatments to keep the homeostasis of ocular surface. Objective. To explore the effect of hypochlorous acid on the meibomian gland dysfunction dry eye through ultrasonic atomization. Methods. We set this study of $0.01 \%$ HOCL and $0.1 \%$ hyaluronate by ultrasonic atomization. All the data was recorded at the $1^{\text {st }}, 15^{\text {th }}, 30^{\text {th }}$, and $55^{\text {th }}$ days. The patients' complains, the meibum analysis, conjunctive congestion, corneal staining, Schirmer's I test, and NIBUT were recorded by K5M, the MMP-9, and IL-2 of tear by inflammation kit; the Demodex was recorded by microscopy. Results. 53 patients have joined this study. There is no statistic difference between them on OSDI (day 15: $p=0.061,30: p=0.055,55: p=0.052$ ); results show the $10.57 \pm 0.13$ and $12.54 \pm 0.17$ reduction on OSDI; the differences of both treatments are significant $\left({ }^{* *} p<0.01\right)$. Increased Schirmer's and TBUT are $3.27 \pm 0.10$ and $6.29 \pm 0.10$ $\left({ }^{* *} p<0.01\right)$ or $7.32 \pm 1.72 \mathrm{~s}$ and $9.22 \pm 1.41 \mathrm{~s}\left({ }^{*} p<0.05\right)$; the decreased conjunctive and corneal staining are $0.23 \pm 0.07$ and $0.45 \pm 0.06\left({ }^{* *} p<0.01\right)$ or $0.42 \pm 0.03$ and $0.37 \pm 0.02\left({ }^{*} p<0.05\right)$ at both groups. The differences of MMP-9 and IL-2 negative rate are significant $\left(Z=0.896,{ }^{* *} p=0.002<0.01 ; Z=0.659,{ }^{* *} p=0.001<0.01\right)$; the number of Demodex mites at first is 10 or 11 , while the last is 2 or $6\left(Z=-4.642,{ }^{* *} p<0.01 ; Z=2.742, p>0.05\right)$. The Demodex count between them is significant $\left(Z=-2.310,{ }^{*} p=0.032<0.05\right)$. The survival times (ST) of each stage at the HOCL are $110.75(108.50 \pm 24.50), 95.50$ $(90.25 \pm 14.50)$, and $75.25(73.48 \pm 8.50)$ min which are shorter than those of control which are $155.50(160.10 \pm 21.50), 130.25$ $(128.25 \pm 16.50)$, and $105.75(102.50 \pm 14.50) \mathrm{min}\left({ }^{* *} p<0.01\right)$. The Demodex eradication rate of HOCL is statistic significant $\left({ }^{*} p 15^{\text {th }}\right.$ vs. $1^{\text {st }}$ day $=0.028<0.05 ;{ }^{* *} p 30^{\text {th }}$ vs. $1^{\text {st }}$ day $=0.002<0.01 ;{ }^{* *} p 55^{\text {th }}$ vs. $1^{\text {st }}$ day $\left.=0.0018<0.01\right)$. Conclusions. $0.01 \%$ HOCL improves the Demodex eradication by shortening the survival time; the HOCL acts on the ocular surface by reducing the inflammation. The ultrasonic atomization helps for the drug usage.

\section{Introduction}

The Dry Eye Work Shop (DEWS) II (2017) report has rewritten the dry eye disease (DED) definition: "Dry eye is a multifactorial ocular surface disease characterized by loss of tear film homeostasis associated ocular symptoms, in which tear film instability and hyperosmolarity, inflammation and ocular surface lesions, as well as neurosensory abnormalities play etiologic roles" [1]. The major prevalence globally ranges from $5-7 \%$ of the USA to $40-70 \%$ of the Eastern Asian [2]. The most vulnerable age of the disease is middle and elderly people (age $>40$ years), but the incidence is rising among the young $[3,4]$. It is crucial for the public to realize the importance of DED by reliable therapy.

Meibomian gland dysfunction (MGD) is defined as a team of aberrant signs, acquired or congenital, implicated 
by dysfunction of the eyelid meibomian glands. MGD leads to affecting tear film function, ocular discomfort, evaporative caused dry eye, or ocular surface problems [5]. The study of 619 randomly chosen participants from a population-based study in north China; $8.6 \%$ were symptomatic MGD while the asymptomatic MGD rate was $21.9 \%$ [6]. Many of these ocular clinical manifestations interweave with dry eye, and Demodex relative MGD is believed to be the key pathological factor to evaporative induced dry eye [7].

Kinds of means are used for the therapy of MGD-related DED, containing nondrug treatments such as Traditional Chinese Medicine (TCM), ultrasonic atomization (UA), intraductal eyelid meibomian gland probing, meibomian gland massage, optimized pulsed light therapy (OPT), and lipiflow. Consider these means are effect to a certain range; difficulties are the inappropriate usage, insignificant effects, high financial expenditure, and the secondary actions [8]. It is necessary for the invention of new proposal to improve the effect.

Hypochlorous acid (HOCL) has multifaceted applications in dermatology, wound healing, eye care, and dentistry. It is the ordinary disinfectant in industrial domestic and medical aspects and has the same active components of household bleach but with a different chemical composition [9]. $\mathrm{HOCl}$ is an attractive material for the nonsynthetic microbial toxic medium. Impurity-free $\mathrm{HOCl}$ originated from the products of the human immune response [10]. Across the oxidative reaction, highly activated molecules such as HOCl are stimulated from the leukocyte's action to external microorganisms [11]. Due to its quickly neutralized feature, HOCL is nontoxic to the ocular surface [12].

Ultrasonic atomization (UA) [13] is a procedure that damages liquid surface tension and atomizes drops into cute elements through ultrasonic vibration. It is the conventional ophthalmological choice in TCM for DED. This procedure of $20 \mathrm{~min}$ makes the droplets fully expose and permeate the ocular surface. Motivation of anatomized steam can accelerate body fluid circulation around eyelid and thus reinforce the release of meibomian gland secretions.

Anyway, concentrate on this earlier work to assess the particular therapeutic safety and effects of ultrasonicatomizer HOCL for the remedy of MGD-related DED which is necessary. Until now, this is the premier double-blind, placebo-controlled randomized study for the hypothesis.

\section{Participants and Evaluations}

2.1. Study Design and Participants. A double-blind layout was designed in this clinical research. Thus, the $1: 1$ ratio of HOCL and placebo treatment groups was carried out to engender random codes using the statistics software SAS9.4 (SAS Institute Inc., Cary, NC, USA), under the block randomization modus. Observed were then followed a medication box labelled with a bunch of number which contained all medicaments. The clinical research group used market-oriented available HOCL $0.01 \%$ pH $5.4 \quad(20 \mathrm{~mL})$ (Avenova ${ }^{\circledR}$, NovaBay Pharmaceuticals, Inc., Emeryville, California, USA) [14]. At the same time, the control was treated with a placebo material $0.1 \%$ Purified Sodium Hyaluronate $(20 \mathrm{~mL})$ (Santen Pharmaceutical Co. Ltd.) which is a $0.72 \%$ $(\mathrm{g} / \mathrm{mL})$ sodium chloride solution with the osmotic pressure of $235 \mathrm{mOsmol} / \mathrm{kg}$ and a $\mathrm{pH}$ value of 6.5 . Patients were also treated with fixed bracket ultrasonic atomizer (product number: HL100A, Yuwell, Jiangsu, China). The medications in this study were atomized for 20 min per 5 days with the ultrasonic atomizer held before the patient's eye. All the patients were treated with meibomian gland massage once a week, 4 times altogether. The treatment duration was 55 days with check point time at the $1^{\text {st }}$ day (baseline) and then the $15^{\text {th }}$ day, $30^{\text {th }}$ day, and $55^{\text {th }}$ day; treatment safety and effectiveness evaluations were executed on-site during the follow-ups. All trails were done on the same instrument. The investigator and the statistician know nothing of patients' identity.

2.2. Inclusion and Exclusion Criteria. All the study subjects were enrolled during Jan 2020-June 2021 period who met the inclusion criteria and were of the same nationality (Han Chinese, aged from 20 to 70 years). Patients with MGD-DED were chosen from those subjects who visited the eye clinic for dry eye.

We excluded patients less than 20 or more than 70 years old; those who with the histories of ocular injury and surgery within 3 months; those with ocular problems such as ocular inflammation, allergy, and nasolacrimal sac problems; those who are using a punctual plug or contact lenses; or those are using eye drops including high-quality artificial tears drops within $24 \mathrm{hr}$ before the examination. Anyone whose outcome results were hard to be confirmed was excluded.

2.3. Ethnic Achievement. This study followed the principium of the Declaration of Helsinki, and this protocol was approved by the institutional review board of the ethics committee of the Affiliated Chenzhou Hospital, Hengyan Medical School, University of South China (ID: 2020YJ03). Informed consent was achieved by the recruiters after a discussion of the purpose and probability on sequences of the clinic trail.

\subsection{Dry Eye Diagnosis and Classification}

(i) The standards for the general normal are as follows $[15,16]$ :

(1) Ocular surface disease index (OSDI) score of less than 12

(2) Without tear film outliers (tear film break-up time, TBUT $>5$ seconds, and Schirmer's test value of $>5 \mathrm{~mm}$ after $5 \mathrm{~min}$ )

(3) Lack of evidence of corneal or conjunctive epithelial erosion with fluorescent staining

(4) Normal lid margins or meibum

(ii) The criteria for the aqueous-deficient dry eye (ADDE) group are as follows $[15,16]$ : 
(1) Presence of dry eye complains (OSDI $\geq 12$ )

(2) Poor tear production as defined by Schirmer's test I $(\leq 5 \mathrm{~mm})$ and tear film instability as named by the $\mathrm{FBUT}(\leq 5$ seconds)

(3) The evidence of corneal or conjunctivae epithelial damage with a fluorescent staining score of $\geq 3$

(iii) The criteria for the MGD group are as follows $[15,16]$ :

(1) Presence of symptoms (OSDI $\geq 12)$

(2) At least one lid margin abnormality

(3) Poor meibomian gland expression (grade $\geq 1$ ) or worse qualitative variety in meibum (meibum quality score $\geq 3$ ).

(iv) The criteria for the ADDE/MGD-related dry eye group are as follows $[15,16]$ :

(1) Presence of dry eye complaints (OSDI $\geq 12$ )

(2) Poor tear production as recorded by Schirmer's test I $(\leq 5 \mathrm{~mm})$ and tear membrane film instability showed by the FBUT( $\leq 5$ seconds)

(3) The evidence of corneal or conjunctivae epithelial discontinue with a fluorescent staining score of $\geq 3$

(4) Not only one lid margin abnormality

(5) Worse meibomian gland expression (grade $\geq 1$ ) or poor quality changes in meibum (meibum qualitative score $\geq 3$ ).

2.5. Ultrasonic Atomization Process [17]. Patients were also assembled with fixed wing ultrasonic nebulizers (product Number: HL100A, Yuwell, Jiangsu, China). The atomizing pipeline was placed $5-10 \mathrm{~cm}$ before the eyelid, and the patients open their eyes larger and stare in all orientations off and on to ensure that the ultrasonic atomizing fine droplets fully penetrate the conjunctive. Treatment fluid was used $20 \mathrm{~mL}$ HOCL or placebo each time. The body temperature of an ultrasonic-atomized aerosol is very close to the room environment; make the entire process arousing the least irritations. The therapy period was 55 days with check points at time day one (baseline) and then at the $15^{\text {th }}$ day, $30^{\text {th }}$ day, and $55^{\text {th }}$ day; treatment safety and efficacy assessments were implemented on-site during these visits.

2.6. Clinic Assessment. One author of our team performed the experiments, and data were obtained from both eyes.
In all the subjects, clinic results were taken sequentially as follows:

Subjective symptoms were graded on a serial scale from 0 to 4 , according to the verified 12-item ocular surface disease index (OSDI) questionnaire. The total OSDI marks range from 0 to 100 and was calculated using the following equation: OSDI $=$ (the summary of scores for every question answered $\times 100) /($ overall number of answered questions $\times 4)$ [18]. K5M:

The following are the objective data through Oculus

(1) Tear meniscus height (TMH) was recorded by Keratograph ${ }^{\circledR}$ 5M (K5M; Oculus, Optikgerate, Germany). The keratograph was set to "film (TF) scan-tear meniscus mode" to capture the image of the TMH of the ocular surface, following the manufacturer's synopsis, as previously reported $[19,20]$

(2) Tear membrane film evaluation with the "TF-Scan, Non-Invasive Keratograph Break-Up Time (NIKBUT) mode" was chosen when the subjects were asked to blink three to five times and then keep their eyes open. There is abnormality on the manifested destructive or break-up of the tear film; meanwhile, the picture was recorded. The equipment provided a representative of tear film break-up all the time, including a tear film topographic map showing the size and location of the tear discontinue regions, as well as the first break time (NIKBUT-first) and the average break-up time (NIKBUT-average; the meanings of all tear film break-up over the entire cornea), as previously described [21]

(3) Conjunctive and corneal staining was graded from 0 to 3 and corresponds to the National Eye Institute (NEI)/Industry Workshop scale [20] from 0 to 33 based on the type of fluorescent staining under the slit lamp

(4) Schirmer's examination I was performed without topical anaesthesia by placing Schirmer's strip in the $1 / 3$ middle lateral part of the lower fornix. The length of wetting was recorded after $5 \mathrm{~min}$, and the patients were asked to make their eyes slightly closed during the test [22]

(5) The eyelid margins and meibomian glands were examined for lid margin anomalous, gland expression, and meibum amount and colour, as previously described [23, 24]. Lid margin anomalous were scored as 0 (absent) or 1 (present) for the following parameters: plugged meibomian gland orifices, vascular congestion, irregularity of the lid margin, and partly expressions of the mucocutaneous borderline $[25,26]$. The extent of meibomian gland expression, using steady digital pressure applied on five glands of the middle third of the lower lid, was graded as such: grade 0 , all five glands expressible; grade 1 , three or four glands expressible; grade 2, one or two glands expressible; and grade 3, no glands expressible [27]. 
The meibum attribute on eight lower lid glands was graded as follows: grade 0 , clear; grade 1, cloudy; grade 2, cloudy with granular particulates; and grade 3 , thick, like toothpaste-like particulates. Each of the eight glands of the lower eyelid was graded on the scale from 0 to 3 . The scores of the eight glands were summarized (range: 0-24) [28]

The following are the data from optical microscopy for Demodex:

(1) For Demodex count [29] on each eye, pluck out 3 upper and lower eyelashes. Try to select the eyelashes with sleeve-like scales at the roots. Clamp the eyelashes with tweezers, and rotate them slightly to loosen the eyelashes. After plucking, place them in parallel on the glass slide. Observe the amount and morphology of Demodex mites under the optical microscope. If the plucked eyelashes are accompanied by scales, add $100 \%$ alcohol or $0.25 \%$ fluorescent sodium solution and observe again. The Demodex count includes its life cycle

(2) For the survival time (ST) of Demodex, written informed protocols have been acquired from each patient before cilia have been removed. After eyelashes have been pulled out from the participants' eyelid in each group at room temperature, these eyelashes with Demodex mites were quickly fastened on the glass slide. HOCL and placebo eyelid patch extracts were then layered onto the glass slide with a micropipette individually. The original sample was treated as the blank origin group. As Demodex is more vulnerable at the young stage of life, only adult Demodex with four pairs of well-developed legs and a robust body were found [30]. After the cilia had been extracted from the eyelid, the activity of the Demodex body and legs was observed instantly and continuously under the ordinary optical microscope at the magnification of $\times 10$ or $\times 40$. The ST was named as the period from the time-point of eyelid patch extract trickled on the body to the cessation of activity

(3) In Demodex mite eradication, only the data of Demodex mites was $\geq 3$; the patient was considered Demodex-positive [30]. Otherwise, "absolute Demodex eradication" was defined as complete Demodex eradication with the tissue reduced to zero [31]

2.7. Tear Film Protein Factors [32]. Tear specimens were obtained using a diagnostic test reagent strip in order to asses with inflammatory kits (Inflammation Dry ${ }^{\circledR}$ test; Rapid Pathogenesis Screening Inc., Sarasota, FL, USA) for each patient. Microbiological pieces were collected before and 20 minutes after implementation of the procedure.

2.8. Sample Size Calculation. There was not a previous study that has directly related studied the subjective complains and objective data with ocular eyelid ultrasonic atomization for disinfection. The arbitrary effect size of $1-\beta 1 / 40.80$ and 0.7 at $\alpha^{1 / 4} 0.05[33,34]$ (a priori, two-tailed, matched-pair test) was selected to count the minimum sample for this study and was estimated to be 25 .

2.9. Statistical Analyses. The main variables did not have a normal distribution; nonparametric tests were used. The clinical variables and ocular surface index were compared between the control and HOCL ultrasonic atomization using the Analysis of Variance (ANOVA) and for categorical variables using Fisher exact or chi-square test. A confrontation of clinical manifestations and ocular surface index is due to the presence of a simultaneous multiple tear membrane film break-up pattern and ocular surface index by the K5M; the observation index does not coincide with the normal distribution and is expressed as the median P50 (P25, P75). Statistical analyses were performed using SPSS (version 19.0; SPSS Inc., Chicago, IL, USA). A $p$ values less than 0.05 was considered statistical significant.

\section{Results and Analysis}

3.1. The Clinic Trail Diagram for the Procedure for the Safety and Efficiency of HOCL and Placebo Drug Ultrasonic Atomization. These 64 recruited patients were randomly selected for double-blind treatments. Two persons in the HOCL and placebo group did not attend screen schedule; accordingly, 30 patients were analyzed in these groups separately. A total of 27 or 26 patients have finished all clinic visits, respectively; this shows that only 1 patient dropped out from each group for adverse events (transient conjunctive hyperaemia) (Figure 1).

3.2. Basic Clinical Information of Enrolled Patients. If the patient wore glasses, the best corrected visual acuity should be recorded, without recording the uncorrected visual acuity. If the patient wore no glasses, the uncorrected visual acuity was recorded. The data of subjects in the designated treatment and amount of subjects in the specified systematization of percentages were based on the number of subjects in the homeostasis treatment. The data in Table 1 show patient clinical idiographic at the beginning. These data show no differentiation in demographic distinctive between them. Results also show no statistic differences at baseline between them in signs of MGD and/or DED as well as in terms of ocular symptoms.

For persistent variables, $p$ value was calculated using Analysis of Variance (ANOVA) and for categorical variables using a chi-square test/Fisher's exact test if cell frequency is $<5$.

\subsection{The Variations of Tear Film Function at the Check Points between HOCL and Placebo Treatment Groups}

3.3.1. Primary Outcomes of Ocular Complaint Scores Slowly Reduced in Both Groups in the following Three Visits (Table 2). There is no statistical significant differentiation between them in the symptom score reduction of the $15^{\text {th }}$ day $(p=0.061)$ and $30^{\text {th }}$ day $(p=0.055)$ and $55^{\text {th }}$ days $(p=0.052)$; results reveal a $10.57 \pm 0.13\left({ }^{* *} p<0.01\right)$ and 


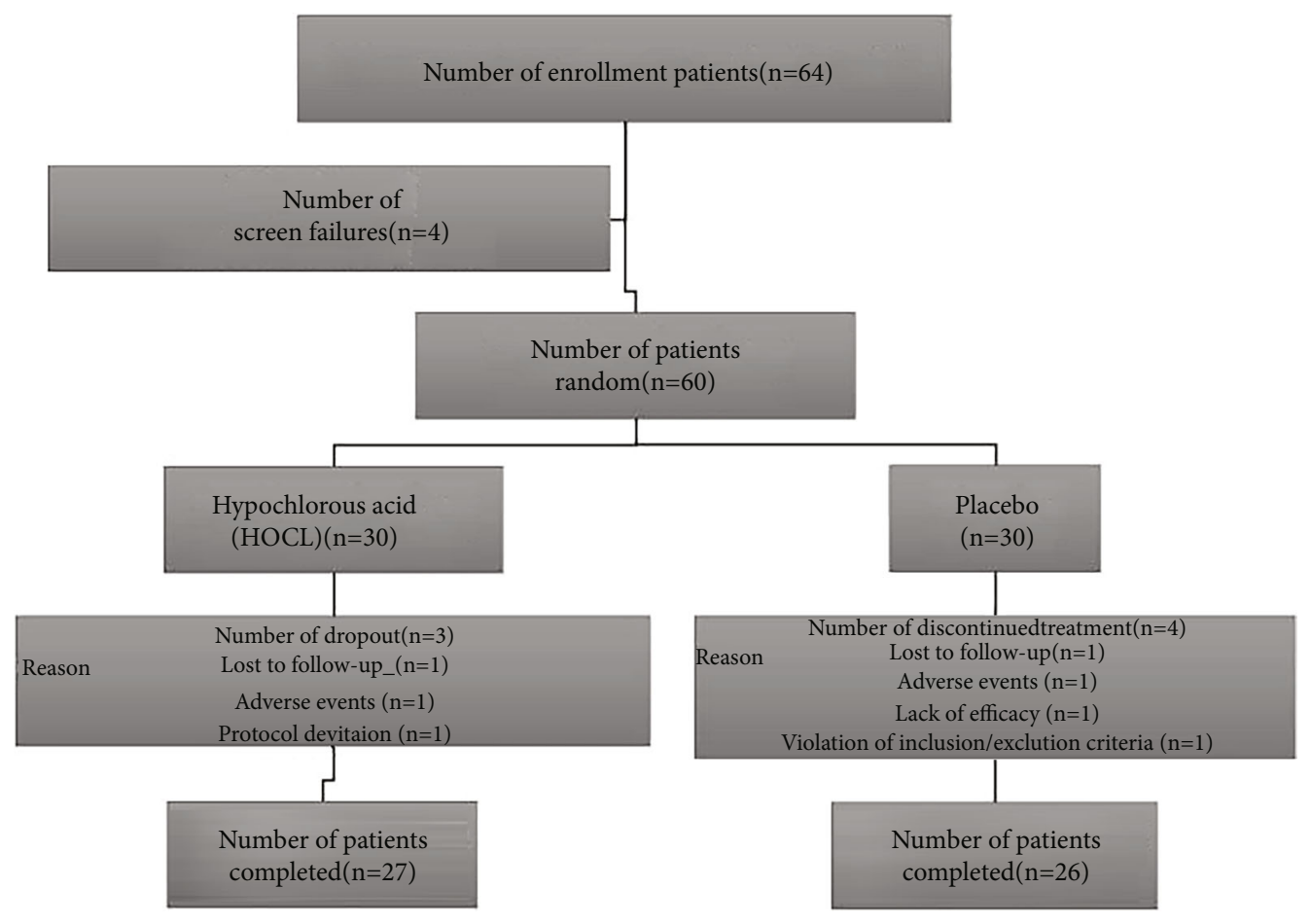

Figure 1: Subject flow chart.

$12.54 \pm 0.17\left({ }^{* *} p<0.01\right)$ decrease in calculated symptom scores after 55 days of atomization management considering the beginnings in both groups, respectively; the difference between them is statistic significant $\left({ }^{* *} p<0.01\right)$ (Table 2). Results for individual complaint score show that the HOCL brings more benefit than that of control.

\subsubsection{Results Show That Both Schirmer's and TBUT Have} Been Ameliorated in Both Groups (Table 2). Tear volume scores (Schirmer's) have increased after the treatment; these increases were $3.27 \pm 0.10$ and $6.29 \pm 0.10$ in the contrast and HOCL groups after 55 days of treatment. There are statistical differences between the HOCL and placebo groups in the increased value (Schirmer's) after 55 days of application $\left.{ }^{* *} p<0.01\right)$ (Table 1). The TBUT in the HOCL were always higher than those of the placebo after therapy over a treatment period. After 55 days of management, TBUT were $7.32 \pm 1.72 \mathrm{~s}$ and $9.22 \pm 1.41 \mathrm{~s}$ in the placebo and HOCL groups, separately. The alterations between both groups are statistical difference $\left({ }^{*} p<0.05\right)$.

3.3.3. Results Show That Conjunctive Congestion Has Been Alleviated by the Atomization Treatment. The decreased values on the conjunctive congestion of the both groups are $0.23 \pm 0.07$ and $0.45 \pm 0.06\left({ }^{* *} p<0.01\right)$ between the beginning and last interviews, respectively (Table 1). The corneal staining has also been decreased after therapy: decreased figures are $0.42 \pm 0.03$ and $0.37 \pm 0.02$ in the placebo contrast and HOCL groups $\left({ }^{*} p<0.05\right)$, respectively. There is a statistical difference in both groups at the final clinical assessment (corneal staining score $>1$ at the beginning) (Table 1).
3.4. The Inflammation Factors of MMP-9 and IL-2 Represent the Ocular Surface Inflammation Reactions

3.4.1. MMP-9 and IL-2 Biomarker. MMP-9 and IL-2 levels were measured at the beginning and last of the study in the tears' components of the recruiter by the Inflammation Dry ${ }^{\circledR}$ test. 16 subjects out of $27(59.26 \%)$ from the HOCL group and 16 subjects out of $26(61.54 \%)$ from the placebo groups demonstrate MMP-9-positive results in the left eye at the $1^{\text {st }}$ day. Three out of $27(11.11 \%)$ from the HOCL group and 12 out of 26 (46.15\%) subjects from the placebo contrast group displayed MMP-9-positive results in the left eye at the $55^{\text {th }}$ day. The MMP-9 shows the inflammation difference between those groups is significant $\left(Z=0.896,{ }^{* *} p=0.002<0.01\right) ; 15$ individuals out of 27 (55.55\%) from the HOCL group and 15 out of $26(57.69 \%)$ from the placebo groups manifest IL-2-positive results in the left eye at the $1^{\text {st }}$ day. Two out of $27(7.41 \%)$ from the HOCL group and 10 out of $26(38.46 \%)$ subjects from the placebo contrast group express IL-2-positive results in the left eye at the $55^{\text {th }}$ day. The IL- 2 shows the inflammation difference between them is significant $\left(Z=0.659,{ }^{* *} p=0.001\right.$ $<0.01)$.

3.5. The Demodex Data. The Demodex detection and calculation through light microscopy between the first and the final examination.

3.5.1. Demodex Count. A total of $27 \mathrm{HOCL}$ treatment patients (54 eyes, 18 females and 9 males, $37.84 \pm 1.02$ years) and 26 placebo individuals (52 eyes, 17 females and 9 males, $38.30 \pm 1.24$ years), matched by gender and age, were 
TABLE 1: Characteristic of the patients at baseline.

\begin{tabular}{|c|c|c|c|c|}
\hline Variables & Hypochlorous acid (HOCL, $N=27$ ) & Placebo $(N=26)$ & Statistical & $p$ value \\
\hline \multicolumn{5}{|l|}{ Demographics } \\
\hline \multicolumn{5}{|l|}{ Age (year) } \\
\hline Mean \pm SEM & $37.84 \pm 1.02$ & $38.30 \pm 1.24$ & $Z=0.1921$ & 0.8343 \\
\hline \multicolumn{5}{|l|}{ Sex } \\
\hline Male, $n(\%)$ & $9(32.29 \%)$ & $9(34.61 \%)$ & & \\
\hline Female, $n(\%)$ & $18(67.71 \%)$ & $17(65.38 \%)$ & $\chi^{2}=0.0583$ & 0.6743 \\
\hline \multicolumn{5}{|c|}{ Best corrected visual acuity } \\
\hline Mean \pm SEM & $0.82 \pm 0.04$ & $0.83 \pm 0.02$ & $Z=-0.6572$ & 0.5692 \\
\hline \multicolumn{5}{|c|}{ Eye symptom score } \\
\hline Mean \pm SEM & $13.38 \pm 0.32$ & $14.17 \pm 0.42$ & $Z=0.2192$ & 0.6172 \\
\hline \multicolumn{5}{|c|}{ Meibum quality score } \\
\hline $0, n(\%)$ & $11(40.83)$ & $10(38.46)$ & $Z=0.4412$ & 0.5658 \\
\hline $1, n(\%)$ & $9(35.33)$ & $8(30.77)$ & & \\
\hline $2, n(\%)$ & $5(21.32)$ & $6(23.08)$ & & \\
\hline $3, n(\%)$ & $2(6.67)$ & $2(7.69)$ & & \\
\hline \multicolumn{5}{|c|}{ Eyelid edge change score } \\
\hline Mean \pm SEM & $1.58 \pm 0.06$ & $1.71 \pm 0.06$ & $Z=-0.0921$ & 0.4835 \\
\hline \multicolumn{5}{|c|}{ Meibum expression score } \\
\hline Mean \pm SEM & $2.43 \pm 0.18$ & $2.65 \pm 0.2$ & $Z=-0.7112$ & 0.5162 \\
\hline \multicolumn{5}{|c|}{ Conjunctive congestion score } \\
\hline $0, n(\%)$ & $7(24.50)$ & $6(23.08)$ & $Z=0.1031$ & 0.8917 \\
\hline $1, n(\%)$ & $12(48.00)$ & $11(42.31)$ & & \\
\hline $2, n(\%)$ & $4(14.81)$ & $7(17.28)$ & & \\
\hline $3, n(\%)$ & $4(14.81)$ & $2(7.69)$ & & \\
\hline \multicolumn{5}{|c|}{ Corneal labeling score } \\
\hline Mean \pm SEM & $0.58 \pm 0.07$ & $0.60 \pm 0.07$ & $Z=-0.4231$ & 0.7325 \\
\hline \multicolumn{5}{|l|}{ Schirmer's I test } \\
\hline Mean \pm SEM & $5.57 \pm 0.31$ & $5.62 \pm 0.18$ & $Z=-0.0347$ & 0.4372 \\
\hline \multicolumn{5}{|c|}{ None interfere tear breakup time(s) } \\
\hline Mean \pm SEM & $4.23 \pm 0.18$ & $4.08 \pm 0.20$ & $Z=1.128$ & 0.2327 \\
\hline
\end{tabular}

consecutively recruited for this study. The medium number of Demodex mites on three eyelashes per patient at the enrollment check of HOCL group for Demodex at the first day is 10 , while the last data of HOCL group is 2 $\left(Z=-4.642,{ }^{* *} p<0.01\right)$. The medium figure of Demodex on three cilia per patient at the enrollment check of the placebo group for Demodex at the first day was 11 , while the last result of placebo group is $6(Z=2.742, p>0.05)$. When we analyze the data of both groups, the difference is statistically significant $\left(Z=-2.310,{ }^{*} p=0.032<0.05\right)$.

3.5.2. The Survival Time (ST) of Demodex. The mean ST of Demodex mite in particular treatments are shown in Figure 2 (survival time of Demodex). The average ST at different check points in the HOCL group is 110.75 $(108.50 \pm 24.50) \mathrm{min}, 95.50(90.25 \pm 14.50) \mathrm{min}$, and 75.25 $(73.48 \pm 8.50)$ min which are significantly lower than the average ST at different check points in the placebo group which are
$155.50(160.10 \pm 21.50) \mathrm{min}, 130.25(128.25 \pm 16.50) \mathrm{min}$, and $105.75(102.50 \pm 14.50) \min \left({ }^{* *} p<0.01\right)$.

3.5.3. The Demodex Mite Eradication Rate. The Demodex counts and ocular parameters at each checkpoint of treatment were also compared (Figure 3 the Demodex mite eradication rate). The Demodex count in the HOCL group has been reduced by $-2.25 \pm 0.84$ after the $15^{\text {th }}$ day's management; meanwhile, the Demodex count in the placebo group has been downregulated by $-0.74 \pm 0.03$ after the $15^{\text {th }}$ day's therapy $(p=0.056>0.05)$. There is significant differentiation in the Demodex mite eradication rate between them at the $55^{\text {th }}$ day $\left({ }^{* *} p=0.006<0.01\right)$. Compared with that of the placebo, the Demodex mite eradication rate of HOCL is statistically significant at each checkpoint $\left({ }^{*} p 15^{\text {th }}\right.$ vs. $1^{\text {st }}$ day $=0.028<0.05 ;^{* *} p 30^{\text {th }}$ vs. $1^{\text {st }} d a y=0.002<0.01 ;^{* *} p 55^{\text {th }}$ vs. $1^{\text {st }}$ day $\left.=0.0018<0.01\right)$. 
TABLE 2: Summary of efficacy endpoints between hypochlorous acid (HOCL) and placebo groups.

\begin{tabular}{|c|c|c|c|}
\hline Visit duration & $\begin{array}{c}\text { HOCL }(n=27), \text { mean } \\
\pm \text { SEM }\end{array}$ & $\begin{array}{c}\text { Placebo }(n=26), \text { mean } \\
\pm \text { SEM }\end{array}$ & $\begin{array}{l}p \text { value by ANOVA for HOCL vs. } \\
\text { placebo }\end{array}$ \\
\hline \multicolumn{4}{|c|}{ (a) Ocular surface disease index (OSDI) scores } \\
\hline $1^{\text {st }}$ day & $18.65 \pm 5.20$ & $21.54 \pm 4.98$ & 0.058 \\
\hline $15^{\text {th }}$ day & $14.25 \pm 4.45$ & $13.68 \pm 5.02$ & 0.061 \\
\hline $30^{\text {th }}$ day & $11.14 \pm 4.14$ & $10.89 \pm 4.98$ & 0.055 \\
\hline $55^{\text {th }}$ day & $8.08 \pm 4.32$ & $9.00 \pm 6.68$ & 0.052 \\
\hline \multicolumn{4}{|c|}{ (b) Schirmer's test average of both eyes } \\
\hline $1^{\text {st }}$ day & $7.09 \pm 2.34$ & $6.65 \pm 1.82$ & 0.411 \\
\hline $15^{\text {th }}$ day & $9.22 \pm 2.12$ & $7.64 \pm 2.04$ & 0.053 \\
\hline $30^{\text {th }}$ day & $12.28 \pm 3.28$ & $9.90 \pm 4.21$ & $<0.05^{*}$ \\
\hline $55^{\text {th }}$ day & $13.38 \pm 2.73$ & $9.92 \pm 2.12$ & $<0.01^{* *}$ \\
\hline \multicolumn{4}{|c|}{$\begin{array}{l}\text { (c) Tear film break-up time (TBUT) average of } \\
\text { both eyes }\end{array}$} \\
\hline $1^{\text {st }}$ day & $4.78 \pm 1.12$ & $4.86 \pm 1.02$ & 0.423 \\
\hline $15^{\text {th }}$ day & $4.89 \pm 1.56$ & $4.97 \pm 1.25$ & 0.351 \\
\hline $30^{\text {th }}$ day & $6.13 \pm 1.13$ & $6.09 \pm 1.08$ & 0.459 \\
\hline $55^{\text {th }}$ day & $9.22 \pm 1.41$ & $7.32 \pm 1.72$ & $<0.05^{*}$ \\
\hline \multicolumn{4}{|c|}{ (d) Conjunctivae labeling average of both eyes } \\
\hline $1^{\text {st }}$ day & $0.86 \pm 0.19$ & $0.88 \pm 0.20$ & 0.677 \\
\hline $55^{\text {th }}$ day & $0.41 \pm 0.27$ & $0.65 \pm 0.25$ & $<0.01^{* *}$ \\
\hline \multicolumn{4}{|c|}{ (e) Corneal labeling average of both eyes } \\
\hline $1^{\text {st }}$ day & $1.05 \pm 0.09$ & $1.24 \pm 0.12$ & 0.406 \\
\hline $55^{\text {th }}$ day & $0.68 \pm 0.11$ & $0.82 \pm 0.09$ & $<0.05^{*}$ \\
\hline
\end{tabular}

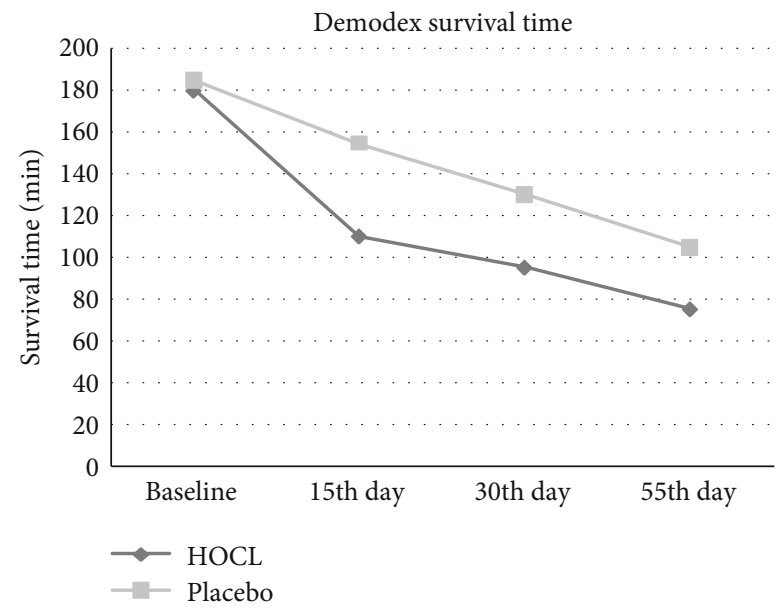

FIgURe 2: The survival time of Demodex.

\section{Discussion}

4.1. The Inflammation of Dry Eye. Dry eye is a chronic recurrent ocular surface disease that most patients' complaints or signs of tear film homeostasis eruption with multiple pathological reactions and the disease or dysfunction of tears fluid producing cells/glands that result in the erratic tear film [35]. Tear eruption is accompanied by raising tear osmotic pressure (local or diffuse area) which induces stress signaling

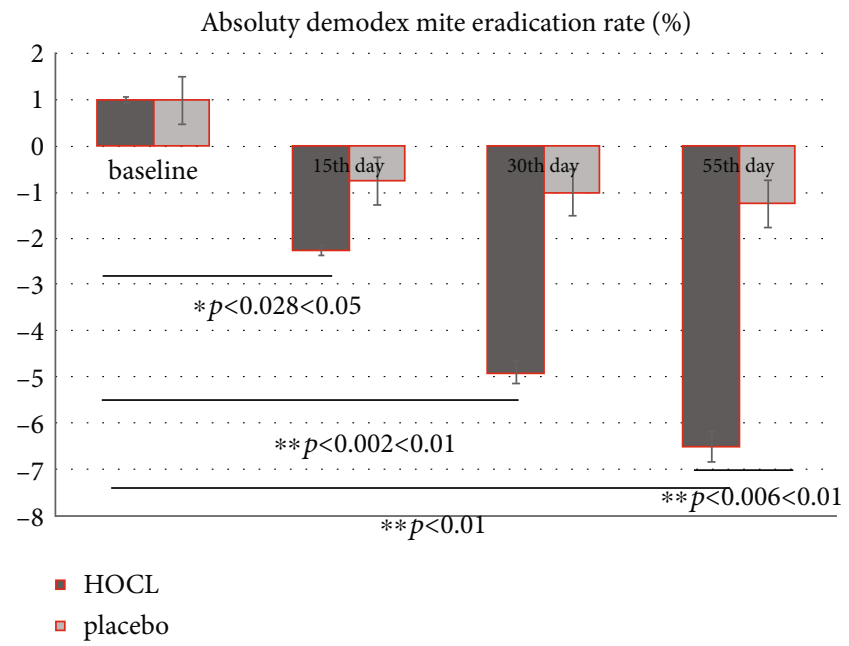

Figure 3: The Demodex mite eradication rate.

pathways in the ocular surface epithelium and resident immunologic cells and triggers the production of innate inflammatory molecules that arouse the vicious selfperpetuating circulation which leads to being further downregulated in tear film function and worse symptoms. Hyperosmolarity stress has been shown to trigger mitogenactivated protein kinase (MAPK) pathway and stimulate secretion of proinflammatory cytokines (e.g., IL-1 $\beta$, IL-2, 
IL-6, and TNF- $\alpha$ ), chemokines, and matrix metalloproteinase such as MMP-3 and MMP-9 as well as cytokine apoptosis [36]. The connection of these inflammatory mediators is intricate which has been shown to affect themselves; the Meibomian gland dysfunction (MGD) "-Relative" dry eye is the leading one, thus amplifying the inflammatory cascade which leads to cornea epithelial barrier disruption, conjunctivae goblet cell loss, and meibomian glandular dysfunction [37]. This manifests the pathological closed loop making the treatment effects uncertain and disease recurrent.

\subsection{The Natural of HOCL to the Ocular Diseases. Pure HOCl} is released as the element of the human immune reaction [32]. During the "oxidative burst," small, highly reactive molecules, such as free $\mathrm{HOCl}$, are generated as leukocyte responds to the pathogenesis of organisms [38]. This element is an oxidant that kills bacteria through the protein and lipid halogenations and/or per oxidation process [39], which has a diffuse spectrum of activity and exhibits rapid killing vitality [40]. The form of HOCL $0.01 \%$ (Avenova ${ }^{\circledR}$, Nova Bay Pharmaceuticals, Inc., Emeryville, California, USA) is commercially formulated free of sodium hypochlorite with the $\mathrm{pH}$ of 3.5-6.0 which has been used in the ocular sickness that has been proved to be safe and effective: Yin et al. and Gold et al. [41, 42] found that $0.01 \%$ HOCL significantly reduced inflammation and was effective in killing $>99.9 \%$ of tested pathogenesis microorganisms without side effect on the ocular surface; HOCL is a potent oxidizing ingredient effective against a wide spectrum of organisms, including the most common bacteria implicated in endophthalmitis after surgery or trauma $[9,32]$. Ngo et al. [43] evaluated the comfort levels of several eyelid cleansing products in the treatment of blepharitis associated with Demodex folliculorum who have achieved the conclusion that the HOCL $0.01 \%$ has the highest degree of patients' comfort.

\subsection{HOCL Can Alleviate the Inflammation Reaction of} Ocular Surface. Ocular inflammations and hyperosmolarity stress are key components of the pathologic circle for the chronic dry eye. Our data show that the ocular inflammation factors between HOCL and placebo group are significantly different $\quad\left(Z=0.896,{ }^{* *} p=0.002<0.01 ; Z=0.659,{ }^{* *} p=\right.$ $0.001<0.01)$ at the end of experiment which confirms with the previous study that HOCL can reduce inflammatory factors, such as decreasing the activity of histamine and interleukin-2 (IL-2) and matrix metalloproteinase-9 (MMP-9) (Table 3) all of which are involved in the development of irritation and itching [44]. HOCL has been shown to have effect on controlling the biofilms and wound healing of ocular epithelium [45]. Some authors [43, 46] have reported the decreasing in the figure of Demodex mites by HOCL. These biological activities are largely due to the per oxidation reaction property. But the traditional use efficiency of HOCL to the ocular surface is limited for its instability inwardness.

4.4. The Mechanism of Ultrasonic Atomization Ocular Surface Device. Ultrasonic atomization moistening device is an extraordinary method for ocular surface which comprises a liquid box and a ventilation high-humidity transparent mirror room around the ocular surface. The atomization system is arranged inside the liquid box, and the atomization system is used for atomizing water in the liquid box. A second gas outlet hole is formed in the liquid box, the first gas inlet hole is formed in the mirror room, and the first gas inlet hole is mixed with the second gas. According to the ultrasonic atomization ocular surface eye moistening device, a high-humidity environment is formed around the ocular surface; the dry eye symptom can be alleviated by the time-depended highly concentrated drugs whose droplets continuously, uniformly, and comprehensively overlay the eyelid, conjunctiva, and cornea and maximize the contact area between the ocular tissue and liquid and there of accelerating drug utilization [47].

4.5. The Clinic Tear Film Data of Ocular Surface. A series of clinical researches have shown that the ultrasonic atomization distinguishing using kinds of liquids improves the meibum expression, increases tear fluid, remits the symptoms and signs of DED, and stabilizes the tear film (Table 2) [47-49]. The placebo contrast in this study was $0.1 \%$ Purified Sodium Hyaluronate. A public study showed that ultrasonic atomization with saline alone can also ameliorate the symptoms and signs (including Schirmer's I test, TBUT, and corneal fluorescent staining). Compared with the artificial tears $(0.1 \%$ Purified Sodium Hyaluronate, Santen Pharmaceutical Co. Ltd.), the treatment effect of the placebo ultrasonic atomization group due to Schirmer's is significantly inferior than that of HOCL $\left({ }^{* *} p<0.01\right)$. The causes for the difference are the chemical nature of HOCL which induces a strong per oxidation and/or halogenation reaction helps for the penetratation ability of eye droplets to the ocular surface. Therefore, in this study, there is statistical difference in the amelioration of TBUT at the endpoint of this trial $\left({ }^{*} p<0.05\right)$ which relates to the significant alleviation effect on the enhancement of oxidizing reactions through the ultrasonic atomization drug deliver system.

4.6. The Pathogenesis Mechanism and the Treatments of Demodex on the MGD-EDE. The Demodex blepharitis has been the hotspot issue by both ophthalmologists and dermatologist in the past decades. Several pathogenesis mechanisms of Demodex blepharitis have been elaborated in previous studies. First, Demodex mites can block the sebaceous ducts and hair follicles mechanically causing epithelial hyperplasia and keratinization, while debris or waste from Demodex mites could diminish innate immune response or the inflammatory reactions. Second, Demodex mites damage the habitat at which they live by persistent adjustment and invasions [50].

Since the Demodex mite is the pathogenesis for blepharitis and Meibomian gland dysfunction, there are kinds of treatments that have been explored. Different tea tree essential oil (TTO) products are now broadly introduced in Demodex blepharitis [51-53]. Liu et al. [50] reported that the okra eyelid dressing effectively eliminated Demodex mites both in vivo and in vitro, whose application was associated with the least ocular discomforts. Besides up-to-data 
TABLE 3: Figures of subjects positive for inflammation biomarker between hypochlorous acid and placebo groups.

\begin{tabular}{|c|c|c|c|c|c|}
\hline \multirow[t]{2}{*}{ Biomarker variation } & \multicolumn{2}{|c|}{$\begin{array}{c}\text { HOCL }(N=27), \text { negative } \\
\text { positive }\end{array}$} & \multicolumn{2}{|c|}{$\begin{array}{c}\text { Placebo }(N=26) \text {, negative } \\
\text { positive }\end{array}$} & \multirow{2}{*}{$\begin{array}{c}p \text { value for HOCL vs. placebo } \\
Z \text {-test }\end{array}$} \\
\hline & $N(\%)$ & $n(\%)$ & $n(\%)$ & $n(\%)$ & \\
\hline \multicolumn{6}{|l|}{ MMP-9 } \\
\hline $1^{\text {st }}$ day & $11(40.74)$ & $16(59.26)$ & $10(38.46)$ & $16(61.54)$ & \\
\hline $55^{\text {th }}$ day & $24(88.88)$ & $3(11.11)$ & $14(53.84)$ & $12(46.15)$ & $Z=0.896,{ }^{* *} p=0.002$ \\
\hline \multicolumn{6}{|l|}{ IL-2 } \\
\hline $1^{\text {st }}$ day & $12(44.44)$ & $15(55.55)$ & $11(42.31)$ & $15(57.69)$ & \\
\hline $55^{\text {th }}$ day & $25(92.59)$ & $2(7.41)$ & $16(61.54)$ & $10(38.46)$ & $Z=0.659,{ }^{* *} p=0.001$ \\
\hline
\end{tabular}

$N$ : number of subjects in designated treatment; $n$ : number of subjects in special category; \%n (number of subjects in special category)/N (number of subjects in designated treatment) $\times 100 ;{ }^{*} p<0.05$ and ${ }^{* *} p<0.01$.

drugs, a previous study also show that the eye care practitioners (ECPs) should additionally consider both $\mathrm{HOCl}-$ based and TTO cleansers as the first-line choice [53]. Murphy et al. [46] have reported a reduction in the amount of Demodex mites with treatment of HOCL which coincides with our study. The treatments for the ocular Demodex are such a hot issue that several clinic research protocols are undergoing; the pure HOCL shows amazing prospect.

In this study, the Demodex count data of the Table 4 has been decreased from 10 to 2 parasites per cilia at the HOCL group $\left(Z=-4.642,{ }^{* *} p<0.01\right)$, from 11 to 6 parasites per cilia at the placebo contrast group $(Z=2.742, p>0.05)$ at the endpoint of this clinic trail that the difference between these groups of the variation is significant $\left(Z=-2.310,{ }^{*} p=0.032<0.05\right)$ which have seemingly slight decrease when making the determination of the clinical improvements. The exact links between the clinical symptoms and parasite burden remain somehow elusive. Gao et al. [51] have introduced this method of Demodex counts with the account of mites recorded in our study, both at the start and at the end of trial. A weekly eyelid scrub with $50 \%$ TTO has been verified successful in killing ocular Demodex infestation, and counts as low as zero have been proved in a previous study after 4 weeks of treatments [52, 53]. This present study reflects that HOCL has the same effect with that of TTO in terms of Demodex eradication on the eyelid tissue; it significantly shortens the survival time of Demodex in vivo, the average ST at different check points in the HOCL group is $110.75(108.50 \pm 31.50) \mathrm{min}, 95.50$ $(90.25 \pm 14.50) \mathrm{min}$, and $75.25(73.48 \pm 8.50) \mathrm{min}$ alone which are significantly shorter compared with the average ST in the placebo group which are $155.50(160.10 \pm 21.50)$ $\min , \quad 130.25 \quad(128.25 \pm 16.50) \quad \mathrm{min}, \quad$ and 105.75 $(102.50 \pm 14.50) \min \left({ }^{* *} p<0.01\right)$ (Figure 2). Therefore, HOCL is proved to be a novel treatment for Demodex blepharitis by shortening the average survival time.

Additionally, compared with the traditional application of $50 \%$ TTO treatment for 3 months in a row, we found that there was no only obvious differentiation between them (Demodex mite eradication rate between the two groups at the $55^{\text {th }}$ day) $\left({ }^{* *} p=0.006<0.01\right)$ (Figure 3$)$ with regard to the average Demodex figures, but the successful killing rate is higher at different check points of this clinic trail in HOCL
TABle 4: The Demodex count between HOCL and placebo groups [P50 (P25, P75)].

\begin{tabular}{lccccc}
\hline Groups & Cases & $1^{\text {st }}$ day & $55^{\text {th }}$ day & $Z$ & $p$ \\
\hline HOCL & 54 & $10(7,15)$ & $2(0,4)$ & -4.642 & $<0.01$ \\
Placebo & 52 & $11(8,18)$ & $6(4,14)$ & 2.742 & $>0.05$ \\
$Z$ & & -0.892 & -2.310 & & \\
$p$ & & 0.431 & $0.032<0.05$ & & \\
\hline
\end{tabular}

groups than that of control $\left({ }^{*} p 15^{\text {th }}\right.$ vs. $1^{\text {st }}$ day $=0.028<0.05$; ${ }^{* *} p 30^{\text {th }}$ vs. $1^{\text {st }}$ day $=0.002<0.01 ;{ }^{* *} p 55^{\text {th }}$ vs. $1^{\text {st }}$ day $=$ $0.0018<0.01$ ) (Figure 3 ). The HOCL is a natural immunity reaction of the human body; our data shows no discomforts which coincide with previous study $[12,14,44,51]$. Otherwise, many discomforts such as allergic reactions, contact dermatitis, and ocular irritations are well-known adverse reactions of TTO proposal [54]. The lower incidence of adverse reactions will ensure higher quality treatment compliance of patient as the reliable choice for MGD-EDE patients.

Due to the limitations of the small quantity of recruit in the present study, the effect of HOCL through ultrasonic atomization for Demodex blepharitis/MGD-DED needs further confirmation in a larger cohort and the observation for the alleviation of MGD-DED symptoms needs a long period. The anti-inflammation effects of the pure HOCL in Demodex blepharitis are elaborated, the mechanisms of the acaricidal effects of HOCL remain to be fully expound. In order to make sure for the symptom alleviation during the chronic disease management process of dry eye, future studies are required to clarify the tenet of HOCL to cure the Demodex blepharitis as well as maintain the ocular surface tear bio film stability.

\section{Conclusion}

We summarized the clinic data that the pure HOCL can improve the eradication rate of the Demodex mite by shortening its average survival time.

The HOCL induces lipid per oxidation reaction to the ocular surface pathogenesis microorganisms that the ocular surface inflammation can be alleviated. 
The ultrasonic atomization drug delivery system helps for the drug usage.

\section{Data Availability}

The data sets used and/or analyzed during the present study are available from the corresponding author on reasonable request.

\section{Consent}

Oral consent from the patient whose lid margin and Demodex photographs score are displayed in Table 1 was obtained prior to publication.

\section{Conflicts of Interest}

The authors declare that they have no competing interests.

\section{Authors' Contributions}

Zhiyuan Li participated in the project design, sample size calculation, and revision of the manuscript. Zhenghua $\mathrm{Li}$ was responsible for the enrolment and follow-up of patients and participated in performing the statistical analysis. Mo Liang, Haiyan Wang, and Yvlian Li performed the clinic treatment and chronic disease management. Xiaoping Zhou and Guoping Kuang also drafted the manuscript. All the authors confirm the authenticity of the raw data and read and approved the final manuscript.

\section{Acknowledgments}

The present study was sponsored by Chenzhou Science and Technology Bureau Fund (grant No. ZDYF-20200092); the hospital management fund by the University of South China (grant No. 2019YJGL04); and the Outstanding Youth Fund of the First People's Hospital of Chenzhou City (grant No. N2019-006). We thank the participants of the study. The authors acknowledge the guidance and support provided by Prof. Hua Wang from the Ophthalmology Department of Xiangya Hosptial, Central South University, Prof. Jundong Zhu from Aier Eye Group, Central South University, and Dr. Jin Liu, Independent Consultant, the Eye Institute of Xiamen University, for conducting this study.

\section{References}

[1] J. P. Craig, J. D. Nelson, D. T. Azar et al., "TFOS DEWS II report executive summary," The Ocular Surface, vol. 15, no. 4, pp. 802-812, 2017.

[2] F. Stapleton, M. Alves, V. Y. Bunya et al., "TFOS DEWS II epidemiology report," The Ocular Surface, vol. 15, no. 3, pp. 334365, 2017.

[3] D. M. Radomska-Leśniewska, A. Osiecka-Iwan, A. Hyc, A. Góźdź, A. M. Dąbrowska, and P. Skopiński, "Therapeutic potential of curcumin in eye diseases," Central European Journal of Immunology, vol. 44, no. 2, pp. 181-189, 2019.
[4] P. K. Gupta, P. Asbell, and J. Sheppard, "Current and future pharmacological therapies for the management of dry eye," Eye \& Contact Lens, vol. 46, no. 2, pp. S64-S69, 2020.

[5] S. Y. Moon, S. A. Han, H. J. Kwon et al., "Effects of lid debris debridement combined with meibomian gland expression on the ocular surface MMP-9 levels and clinical outcomes in moderate and severe meibomian gland dysfunction," $B M C$ Ophthalmology, vol. 21, no. 1, p. 175, 2021.

[6] J. W. Hu, S. Y. Pan, H. Yang, and X. H. Xiao, "Prevalence and risk factors of dry eye disease in young and middle-aged office employee: a Xi'an study," International Journal of Ophthalmology, vol. 14, no. 4, pp. 567-573, 2021.

[7] J. Xiao, M. Y. Adil, X. Chen et al., "Functional and morphological evaluation of meibomian glands in the assessment of meibomian gland dysfunction subtype and severity," American Journal of Ophthalmology, vol. 209, pp. 160-167, 2020.

[8] L. Jie, O. Shang-Kun, L. Wei, L. Zu-Guo, and P. Qing-Hua, "Physical therapy modalities of Western medicine and traditional Chinese medicine for meibomian gland dysfunction," Digital Chinese Medicine., vol. 3, no. 4, pp. 229-238, 2020.

[9] E. Romanowski, N. Stella, K. Yates, K. M. Brothers, R. P. Kowalski, and R. M. Q. Shanks, "In vitro evaluation of a hypochlorous acid hygiene solution on established biofilms," Eye \& Contact Lens, vol. 44, no. 2, pp. S187-S191, 2018.

[10] I. A. Jones and L. T. Joshi, "Biocide use in the antimicrobial era: a review," Molecules, vol. 26, no. 8, p. 2276, 2021.

[11] A. Ulfig and L. I. Leichert, "The effects of neutrophil-generated hypochlorous acid and other hypohalous acids on host and pathogens," Cellular and Molecular Life Sciences, vol. 78, no. 2, pp. 385-414, 2021.

[12] W.-H. Chang, P.-Y. Liu, M.-H. Lin et al., "Applications of hyaluronic acid in ophthalmology and contact lenses," Molecules, vol. 26, no. 9, p. 2485, 2021.

[13] Z. Haoyan and Z. Tao, "Clinical observation of ultrasonic atomization of traditional Chinese medicine in treatment of dry eye in patients with type 2 diabetes mellitus," Clinical Journal of Traditional Chinese Medicine, vol. 4, pp. 743-747, 2020.

[14] A. Fam, P. T. Finger, A. S. Tomar, G. Garg, and K. J. Chin, "Hypochlorous acid antiseptic washout improves patient comfort after intravitreal injection: A patient reported outcomes study," Indian Journal of Ophthalmology, vol. 68, no. 11, pp. 2439-2444, 2020.

[15] R. Arita, N. Morishige, S. Koh et al., "Increased tear fluid production as a compensatory response to meibomian gland loss: a multicenter cross-sectional study," Ophthalmology, vol. 122, no. 5, pp. 925-933, 2015.

[16] J. W. Jung, J. Y. Kim, H. S. Chin, Y. J. Suh, T. I. Kim, and K. Y. Seo, "Assessment of meibomian glands and tear film in postrefractive surgery patients," Clinical \& Experimental Ophthalmology, vol. 45, no. 9, pp. 857-866, 2017.

[17] Z. Liu, M. Jin, Y. Li et al., "Efficacy and safety of Houttuynia eye drops atomization treatment for meibomian gland dysfunction-related dry eye disease: a randomized, doubleblinded, placebo-controlled clinical trial," Journal of Clinical Medicine, vol. 9, no. 12, p. 4022, 2020.

[18] R. M. Schiffman, M. D. Christianson, G. Jacobsen, J. D. Hirsch, and B. L. Reis, "Reliability and validity of the ocular surface disease index," Archives of Ophthalmology, vol. 118, no. 5, p. $615,2000$.

[19] P. Arriola-Villalobos, J. I. Fernández-Vigo, D. Díaz-Valle, J. E. Peraza-Nieves, C. Fernández-Pérez, and J. M. Benítez-del- 
Castillo, "Assessment of lower tear meniscus measurements obtained with Keratograph and agreement with Fourierdomain optical-coherence tomography," The British Journal of Ophthalmology, vol. 99, no. 8, pp. 1120-1125, 2015.

[20] K. W. Lee, J. Y. Kim, H. S. Chin, K. Y. Seo, T. I. Kim, and J. W. Jung, "Assessment of the tear meniscus by strip meniscometry and keratograph in patients with dry eye disease according to the presence of meibomian gland dysfunction," Cornea, vol. 36, no. 2, pp. 189-195, 2017.

[21] J. Kim, J. Y. Kim, K. Y. Seo, H. S. Chin, J. W. Jung, and J. W. Jung, "Location and pattern of non-invasive keratographic tear film break-up according to dry eye disease subtypes," Acta Ophthalmologica, vol. 97, no. 8, pp. e1089-e1097, 2019.

[22] N. Li, X. G. Deng, and M. F. He, "Comparison of the Schirmer I test with and without topical anesthesia for diagnosing dry eye," International Journal of Ophthalmology, vol. 5, no. 4, pp. $478-481,2012$.

[23] S. C. Pflugfelder, S. C. Tseng, O. Sanabria et al., "Evaluation of subjective assessments and objective diagnostic tests for diagnosing tear-film disorders known to cause ocular irritation," Cornea, vol. 17, no. 1, pp. 38-56, 1998.

[24] C. H. Yeh, S. X. Yu, and M. C. Lin, "Meibography phenotyping and classification from unsupervised discriminative feature learning," Translational Vision Science \& Technology, vol. 10, no. 2, p. 4, 2021.

[25] K. K. Nichols, G. N. Foulks, A. J. Bron et al., "The international workshop on meibomian gland dysfunction: executive summary," Investigative Ophthalmology \& Visual Science, vol. 52, no. 4, pp. 1922-1929, 2011.

[26] J. E. Kim, N. R. Kim, H. S. Chin, K. Y. Seo, T. I. Kim, and J. W. Jung, "Factors associated with ocular surface epithelial damage in patients with primary Sjögren's syndrome," BMC Ophthalmology, vol. 21, no. 1, p. 114, 2021.

[27] S. Y. Lee, K. Lee, C. K. Park et al., "Meibomian gland dropout rate as a method to assess meibomian gland morphologic changes during use of preservative-containing or preservative-free topical prostaglandin analogues," PLoS One, vol. 14, no. 6, article e0218886, 2019.

[28] K. Y. Seo, S. M. Kang, D. Y. Ha, H. S. Chin, and J. W. Jung, "Long-term effects of intense pulsed light treatment on the ocular surface in patients with rosacea-associated meibomian gland dysfunction," Contact Lens \& Anterior Eye, vol. 41, no. 5, pp. 430-435, 2018.

[29] Chinese branch of Asian dry eye association, ophthalmology group of ophthalmology committee of cross-strait medical exchange association, "Consensus of diagnosis and treatment experts on vermicoid mite blepharitis in China 2018," Chinese Journal of Ophthalmology, vol. 54, no. 7, pp. 491-495, 2018.

[30] Y. Y. Gao, M. A. di Pascuale, W. Li et al., "High prevalence of Demodex in eyelashes with cylindrical dandruff," Investigative Ophthalmology \& Visual Science, vol. 46, no. 9, pp. 3089-3094, 2005.

[31] X. Zhang, N. Song, and L. Gong, "Therapeutic effect of intense pulsed light on ocular demodicosis," Current Eye Research, vol. 44, no. 3, pp. 250-256, 2019.

[32] D. W. Stroman, K. Mintun, A. B. Epstein et al., "Reduction in bacterial load using hypochlorous acid hygiene solution on ocular skin," Clinical Ophthalmology, vol. 11, no. 11, pp. 707-714, 2017.

[33] S. B. Hulley, S. R. Cummings, W. S. Browner, D. G. Grady, and T. B. Newman, Designing Clinical Research: An Epidemiologic
Approach, Lippincott Williams\& Wilkins, Philadelphia, PA, USA, 4th edition, 2013.

[34] F. Faul, E. Erdfelder, A. G. Lang, and A. Buchner, "G* power 3: a flexible statistical power analysis program for the social, behavioral, and biomedical sciences," Behavior Research Methods, vol. 39, no. 2, pp. 175-191, 2007.

[35] P. Aragona, G. Giannaccare, R. Mencucci, P. Rubino, E. Cantera, and M. Rolando, "Modern approach to the treatment of dry eye, a complex multifactorial disease: a P.I.C.a.S.S.O. board review," British Journal of Ophthalmology, vol. 105, no. 4, pp. 446-453, 2021.

[36] S. C. Pflugfelder and C. S. de Paiva, "The pathophysiology of dry eye disease: what we know and future directions for research," Ophthalmology, vol. 124, no. 11, pp. S4-S13, 2017.

[37] T. Yamaguchi, "Inflammatory Response in Dry Eye," Investigative Ophthalmology \& Visual Science, vol. 59, no. 14, p. DES192, 2018.

[38] M. Jaganjac, A. Cipak, R. J. Schaur, and N. Zarkovic, "Pathophysiology of neutrophil-mediated extracellular redox reactions," Frontiers in Bioscience-Landmark., vol. 21, no. 4, pp. 839-855, 2016.

[39] M. J. Davies and C. L. Hawkins, "The role of Myeloperoxidase in biomolecule modification, chronic inflammation, and disease," Antioxidants \& Redox Signaling, vol. 32, no. 13, pp. 957-981, 2020.

[40] T. Appiah, Y. D. Boakye, and C. Agyare, "Antimicrobial activities and time-kill kinetics of extracts of selected Ghanaian mushrooms," Evidence-based Complementary and Alternative Medicine, vol. 2017, Article ID 4534350, 15 pages, 2017.

[41] H. Y. Yin, S. Tighe, S. C. Tseng, and A. M. S. Cheng, "Successful management of chronic blepharo-rosacea associated demodex by lid scrub with terpinen-4-ol," American Journal of Ophthalmology Case Reports, vol. 23, article 101171, 2021.

[42] M. H. Gold, A. Andriessen, A. C. Bhatia et al., "Topical stabilized hypochlorous acid: the future gold standard for wound care and scar management in dermatologic and plastic surgery procedures," Journal of Cosmetic Dermatology, vol. 19, no. 2, pp. 270-277, 2020.

[43] W. Ngo, L. Jones, and E. Bitton, "Short-term comfort responses associated with the use of eyelid cleansing products to manage Demodex folliculorum," Eye \& Contact Lens, vol. 44, no. 2, pp. S87-S92, 2018.

[44] J. Q. Del Rosso and N. Bhatia, "Status report on topical hypochlorous acid: clinical relevance of specific formulations, potential modes of action, and study outcomes," The Journal of Clinical and Aesthetic Dermatology, vol. 11, no. 11, pp. 3639, 2018.

[45] M. M. Kiamco, H. M. Zmuda, A. Mohamed et al., "Hypochlorous-acid-generating electrochemical scaffold for treatment of wound biofilms," Scientific Reports, vol. 9, no. 1, p. 2683, 2019.

[46] O. Murphy, V. O'Dwyer, and A. Lloyd-McKernan, “The efficacy of tea tree face wash, 1, 2-Octanediol and microblepharoexfoliation in treating _Demodex folliculorum_blepharitis," Contact Lens \& Anterior Eye, vol. 41, no. 1, pp. 77-82, 2018.

[47] K. Ma, Q. S. Li, Z. Y. Zhang, M. H. Xiang, and Y. Q. Zhao, "Research progress in physical therapy for dry eye," International Eye Science, vol. 18, pp. 660-663, 2018.

[48] J. C. Yuqiu Xu, "Research progress of ultrasonic atomization treating dry eye," Journal Traditional Chinese Medicine Ophthalmology, vol. 30, pp. 367-370, 2020. 
[49] Y. Zhou, "Clinical observation and analysis of dry eye after phacoemulsification and intraocular lens implantation with traditional Chinese medicine ultrasonic atomization," Hunan University of Chinese Medicine, vol. A01, pp. 45-46, 2018.

[50] W. Liu and L. Gong, "Anti-demodectic effects of okra eyelid patch inDemodexblepharitis compared with tea tree oil," Experimental and Therapeutic Medicine, vol. 21, no. 4, p. 338, 2021.

[51] Y. Y. Gao, M. A. Di Pascuale, A. Elizondo, and S. C. Tseng, "Clinical treatment of ocular demodecosis by lid scrub with tea tree oil," Cornea, vol. 26, no. 2, pp. 136-143, 2007.

[52] S. Tighe, Y. Y. Gao, and S. C. Tseng, "Terpinen-4-ol is the most active ingredient of tea tree oil to kill Demodex mites," Translational Vision Science \& Technology, vol. 2, no. 7, p. 2, 2013.

[53] E. Bitton, Treatment of Demodex with hypochlorous acid: case report consistent treatment with hypochlorous acid may improve symptoms for symptomatic dry eye patients. Dry eye patients report improvement in symptoms with hypochlorous acid use over 30 days. This abstract was presented at the 2019 ARVO annual meeting, Vancouver, Canada, 2019.

[54] H. Koo, T. H. Kim, K. W. Kim, Y. S. Chun, J. C. Kim, and J. C. Kim, "Ocular surface discomfort and Demodex: effect of tea tree oil eyelid scrub in Demodex blepharitis," Journal of Korean Medical Science, vol. 27, no. 12, pp. 1574-1579, 2012. 